\title{
Cytopath: Simulation based inference of differentiation trajectories from RNA velocity fields
}

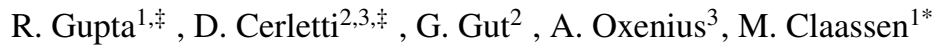 \\ 1 Internal Medicine I, University Hospital Tübingen, Faculty of Medicine, University of Tübingen, Otfried-Müller- \\ Straße 10, Tübingen, Germany. \\ 2 Institute of Molecular Systems Biology, ETH Zurich, Otto-Stern-Weg 3, 8093 Zürich, Switzerland. \\ 3 Institute of Microbiology, ETH Zurich, Vladimir-Prelog-Weg 4, 8093 Zürich, Switzerland. \\ †shared contribution \\ *Corresponding author: manfred.claassen@med.uni-tuebingen.de
}

\begin{abstract}
Unsupervised trajectory inference from single cell RNA sequencing data bears the potential to systematically reconstruct complex differentiation processes but remains challenging notwithstanding the many available solutions. In general, trajectory and pseudotime inference methods have so far suffered from the ambiguity of the static single-cell transcriptome snapshots lacking a measure of directionality of transcriptional activity. We report Cytopath, a method for trajectory inference that takes advantage of transcriptional activity information from RNA velocity of single cells to perform trajectory inference. Cytopath performs this task by defining a Markov chain model, simulation of an ensemble of possible differentiation trajectories and subsequent statistical analysis to extract the topological and molecular characteristics of the studied differentiation process. We demonstrate the capability of Cytopath to reconstruct differentiation trajectories with varying bifurcated and circular topology studied in single-snapshot as well as time-series single-cell RNA sequencing experiments. Comparison to state-of-the art trajectory inference approaches demonstrate superior and enabling capability to reconstruct the considered differentiation trajectories. Trajectory inference constitutes a frequent step in interpreting single-cell RNA sequencing studies. We expect Cytopath to enable researchers to tap the directionality information present in single-cell RNA sequencing data to achieve trajectory inference for possibly complex lineages at an unprecedented precision and resolution.
\end{abstract}

Keywords Trajectory inference $\cdot$ RNA velocity $\cdot$ Single cell RNA sequencing

\section{Introduction}

Biological processes such as neuronal cell type differentiation [Manno et al., 2018], pancreatic endocrinogenesis [Byrnes et al., 2018] and immune response to viral infection [Cerletti et al., 2020] are defined by a temporal sequence of coordinated phenotypic state changes in the context of, possibly heterogeneous, cell populations. Such phenotypic states can be characterized by e.g. epigenetic, transcriptional and proteomic cell profiles. Furthermore, biological processes are often asynchronously triggered and might give rise to bi- and multifurcated state sequences.

This situation requires single-cell approaches to measure and ultimately investigate these biological processes. The repertoire of suitable technologies to monitor different types of molecular profiles has increased dramatically over the last years. In particular single-cell RNA sequencing (scRNAseq) has gained in wide spread use due to the broad applicability of sequencing technology. While these measurements are information rich, their analysis and interpretation is challenged by high-dimensionality, measurement noise and destructive nature only yielding snapshots of the whole process.

Different computational approaches have been proposed to reconstruct cell state sequences of biological processes from scRNAseq data. This reconstruction involves a computational inference step, also known as pseudotime ordering [Tritschler et al., 2019]. This procedure aims at ordering the measured molecular profiles consistent with the state sequence of the underlying process and is typically guided by the assumption that phenotypic similarity reflects temporal proximity and therefore utilize similarity of gene expression profiles to estimate adjacency in a graph of cell state transitions. Various statistical approaches have been developed to address this task with varying amount of success depending on the size and topology of the data. The interpretation of pseudotime ordering for branched processes is particularly challenging and requires an additional trajectory inference step. A comprehensive review by [Saelens et al., 2019] on 
the performance of various methods on multiple topologies suggests, Slingshot [Street et al., 2018] has the best general performance on datasets with multiple branching lineages, and Monocle3 [Trapnell et al., 2014], as a popular runner-up for pseudotime inference that does not produce explicit trajectories per lineage.

Static expression profiles are ambiguous with respect to the directionality of potential cell state transitions. This ambiguity constitutes a major limitation of pseudotime ordering and trajectory inference, and specifically precludes data driven assignment of start and endpoints without previous knowledge about the process and the detection of cell state transitions marked by large deviations in expression patterns. Recently, it became possible to estimate transcriptional activity from scRNAseq data via RNA velocity analysis [Manno et al., 2018] consequently allowing to infer likely transitions between different cell states in a data-driven fashion, ultimately opening the possibility to mitigate the limitations of afore reconstruction approaches.

So far trajectory inference approaches lack the incorporation of such directionality information to estimate pseudotime or construct trajectories. In this work we aim at closing this gap by proposing Cytopath, a simulation based trajectory inference approach that accounts for RNA velocity. We show that Cytopath achieves accurate and robust cell state trajectories of known biological process with linear, circular and bifurcated topologies in datasets and is superior to state-of-the art trajectory inference approaches.

\section{Results}

In this section we present the core features and mechanisms of Cytopath. Trajectory inference is performed on three scRNA seq. datasets presenting three different scenarios. We show that Cytopath successfully recovers known biological processes in the datasets with high accuracy. Furthermore we compare the performance of Cytopath to widely used trajectory inference tools, Slingshot and Monocle3. We demonstrate that the shortcomings of these tools which do not include RNA velocity information are successfully addressed by Cytopath.

\section{Simulation based trajectory inference with Cytopath}

Trajectory inference with Cytopath follows the RNA velocity analysis of a scRNA seq dataset, and is specifically based on the resulting cell-to-cell transition probability matrix. The objective of trajectory inference with Cytopath is to estimate trajectories from root to terminal cell states, defining the start and end points of the studied differentiation process respectively. Root and terminal states can either be derived from a Markov random-walk model utilizing the transition probability matrix itself, as described in [Manno et al., 2018] or can be supplied by the user based on suitable prior knowledge.

The trajectory inference process is divided into four steps [Figure 1]. In the first step, Markov sampling of consecutive cell state transitions is performed based on the probabilities of the transition matrix, resulting in an ensemble of simulated cell state sequences. Sampling is initialized at the predefined root states. Sampling is performed for a fixed number of steps until a sufficient number of unique cell state sequences reaching the terminal states have been generated [Figure $1 \mathrm{~B}-\mathrm{C}]$.

The generated cell state sequences are individual simulations of the biological process from the root to the terminal state. Due to the stochastic nature of the sampling process, the cell state sequences cannot be considered as aligned with respect to the cell states at each transition step. Consequently, in the second step, simulations that terminate at a common terminal state are aligned using Dynamic Time Warping. The procedure aligns simulations to a common differentiation coordinate such that cell states from any simulation at a particular differentiation coordinate (pseudotime) represent similar cell states [Figure[1C-D].

In the third step, consensus states across the steps of the aligned simulations are estimated, giving rise to the reported trajectory. Cell states at every step of the ensemble of aligned simulations are averaged and the average value is considered as the consensus state of the trajectory at the particular step. Alternatively, trajectories can be anchored to observed cell states in the data by choosing the cell state closest to the aforementioned average value.

In the final step, cell groups are assigned to each step of the inferred trajectory. Assignment is based on an alignment score evaluating for each cell both similarity of its static as well as the velocity profile with those of the consensus states. The score is used to measure the trajectory position as well as the relative association of a cell state to possibly multiple branches of a differentiation processes with complex topology. 


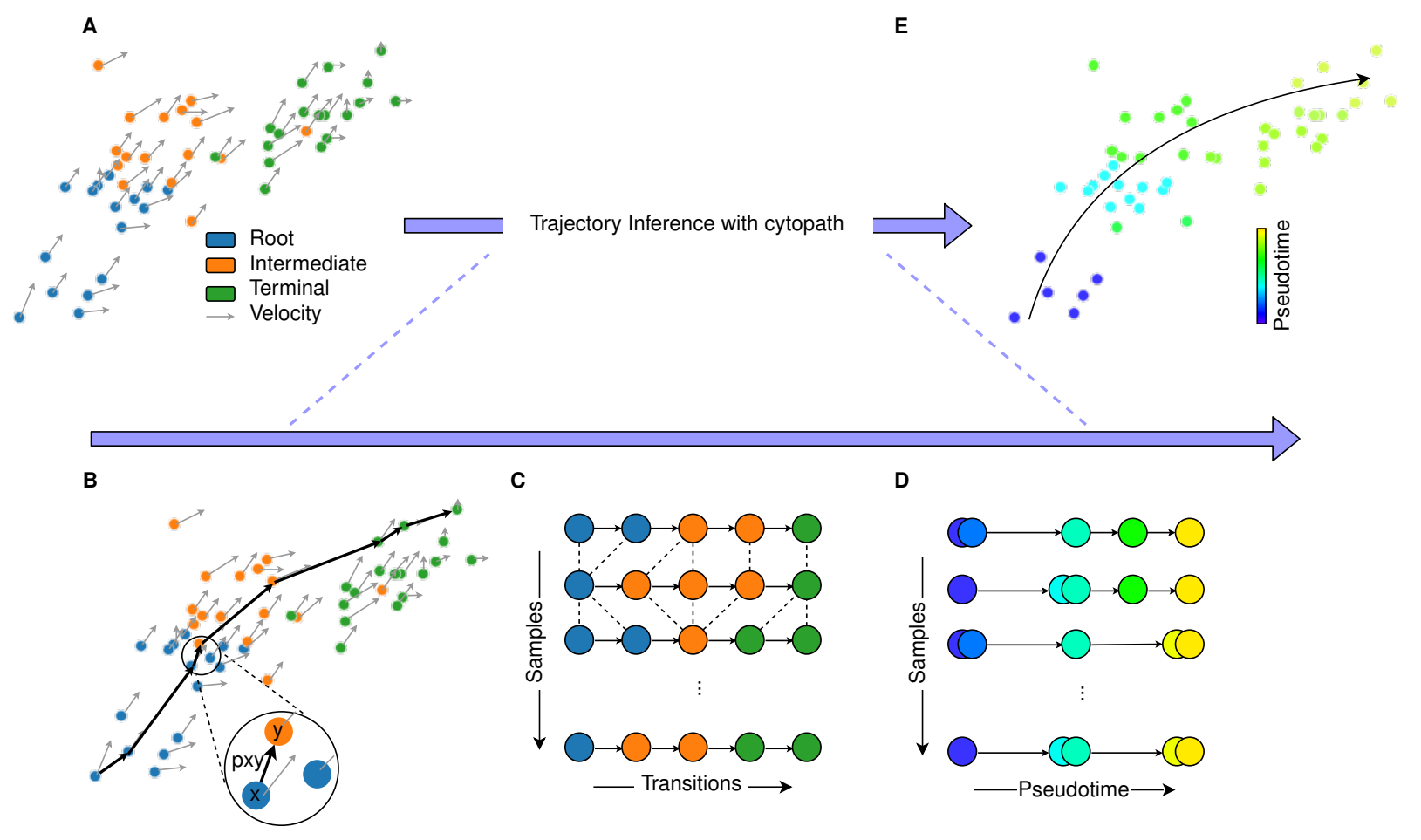

Figure 1: Cytopath schematic. (A) Input data for trajectory inference consisting of single cell gene expression profiles, RNA velocity profiles, root and terminal state annotation. (B) Simulation of the differentiation process by sampling a markov chain is performed based on the cell to cell transition probabilities. Sampling is initialized on cells from a RNA velocity derived root state. (C) Simulation is performed for a defined number of steps. Transition steps are aligned using Dynamic Time warping. (D) After alignment cells at each transition step represent the same consensus state. (E) Cells along the inferred trajectory are assigned the aligned transition-step value as the pseudotime.

\section{Reconstruction of neuronal differentiation in the developing mouse hippocampus}

We assessed the capability to reconstruct developmental processes with multiple branching. To this end, we applied Cytopath, Monocle3 and Slingshot to the developing mouse hippocampus dataset, which was first used to demonstrate RNA velocity of single cells. This dataset is composed of 18,140 cells. RNA velocity was used to identify the root and terminal regions [Manno et al., 2018].

Data from the original publication including t-SNE projection, RNA velocity and transition probability matrix were utilized to for trajectory inference with Cytopath. Spearman correlation of inferred trajectory orderings with the cell type identities and their ordering reported in the initial study [Figure 2 $\mathrm{A}$ ] were used for trajectory inference performance assessment [Figure 2B]. We also supply the known root and terminal states as supervision to Slingshot and Monocle3 (root states only). The dataset comprises five terminal regions and a common root state. The topology of the data is multifurcating with development branches arising directly from the root state, namely Astrocytes and Oligodendrocyte precursors (OPC), but also as branches from intermediate states, namely Neuroblast and Cajal-Retzius differentiation.

Trajectories and pseudotime estimated by each trajectory inference method are shown in Figure 2C-E. Cytopath estimates the correct number of trajectories to each terminal state while Slingshot produces a spurious trajectory despite having the terminal states as supervision. Monocle 3 produces two parallel trajectories within the Granulocyte branch, wrongly dividing into smaller parts. Monocle3 further fails to produce a connected trajectory, producing a disjoint graph and therefore unable to estimate pseudotime for a large portion of the dataset.

The trajectory inference procedure was performed ten times for each method. Spearman correlation between the pseudotime estimated by each method and the true lineage ordering of cell identities shown indicates that pseudotime estimated by Cytopath outperforms other methods in terms of recovering the expected ordering of cells for OPC. The correlation distribution for Cytopath per lineage has much lower variance compared to Slingshot and Monocle3 [Figure 2B]. 

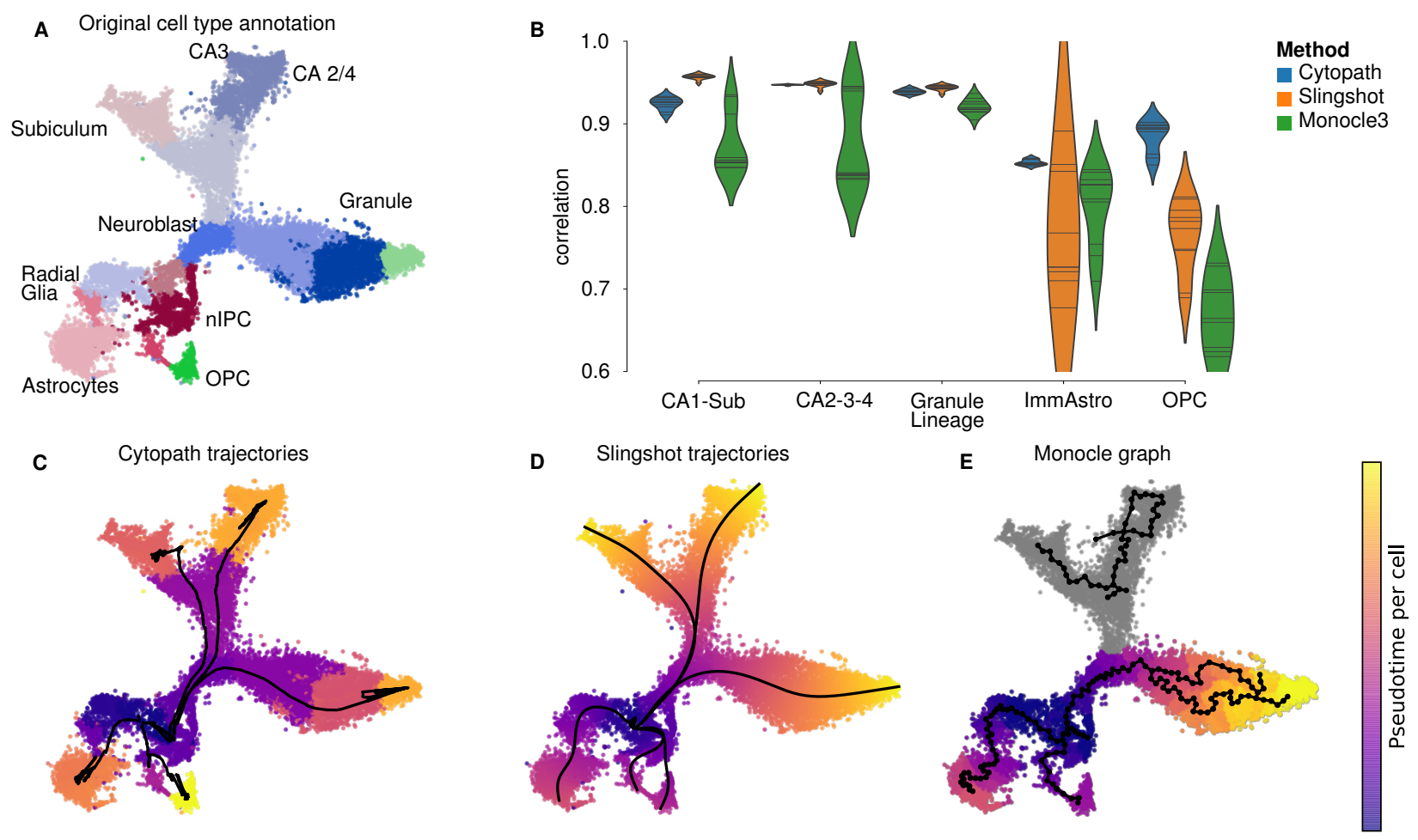

Figure 2: Reconstruction of neuronal differentiation in the developing mouse hippocampus. (A) t-SNE projection of the dendate gyrus scRNAseq dataset annotated with stages of neuronal differentiation. (B) Spearman correlation of pseudotime values assigned by each method to the known ordering of cell types per trajectory. (C-E) Trajectories inferred and mean pseudotime per cell by Cytopath (C), Slingshot (D) Monocle3 (E).

\section{Reconstruction of interlaced cell cycling and bifurcated differentiation in pancreatic endocrinogenesis}

We further assessed trajectory inference performance for processes with multiple interlaced non-trivial topologies. To this end we considered a dataset studying pancreatic endocrinogenesis with lineages to four terminal states, alpha, beta, gamma and delta cells and dominant cell cycling at the onset of differentiation. [Byrnes et al., 2018]. Preprocessing, RNA velocity and the transition probability matrix estimation were performed with scvelo [Bergen et al., 2020].

Biological cell type annotation from [Byrnes et al., 2018] was used to provide root and terminal state supervision to Cytopath [Figure $3 \mathrm{~A}$ ]. Trajectories estimated by cytopath to each terminal state capture the expected differentiation events of endocrinogenesis [Figure 3B]. [Byrnes et al., 2018]

Cell cycle scoring of cells in the root region revealed clearly distinct cell cycle states [Figure $3 \mathrm{C}$ ]. The structure of the mean trajectory estimated by the baseline approaches in the root region of this dataset does not adher to the cyclic structure of the cell cycle stages [3D-F]. In contrast, the trajectory inferred by cytopath from the ensemble of XY simulations recapitulates the circular structure of the cell cycle. We further assessed the frequency of cell cycle phase as a function of the transition step of individual simulations comprising the final trajectory [Figure 3G]. Spearman correlation of cell cycle phase with the transition steps of each simulation indicate faithful recapitulation of the cell cycle stages at the single-simulation level [Figure $3 \mathrm{H}$ ].

The simulation based approach of Cytopath ensures that even in the absence of explicit supervision, cyclic transcriptional patterns are faithfully reconstructed. In contrast, due to the absence of RNA velocity information, the designated root states appear to be isotropic for conventional trajectory inference approaches like Slinghshot and Monocle3 and therefore are unable to capture structured transcriptional heterogeneity within this region.

In Figure $\mathrm{S} 2$ we show the trajectory estimation with respect to the full pancreatic endocrinogenesis process. Slingshot and Monocle 3 produce spurious or to few trajectories respectively. 
A

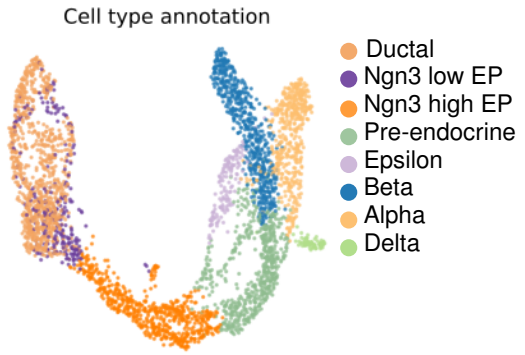

B

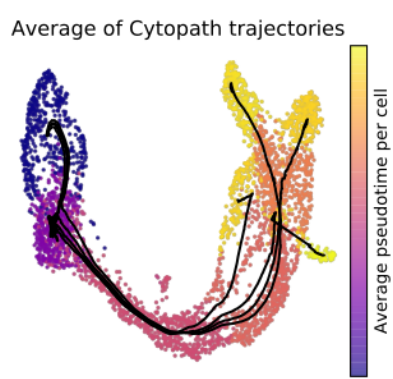

D Average of Cytopath trajectories

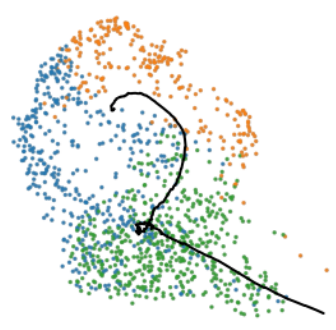

G Cell cycle phase distribution

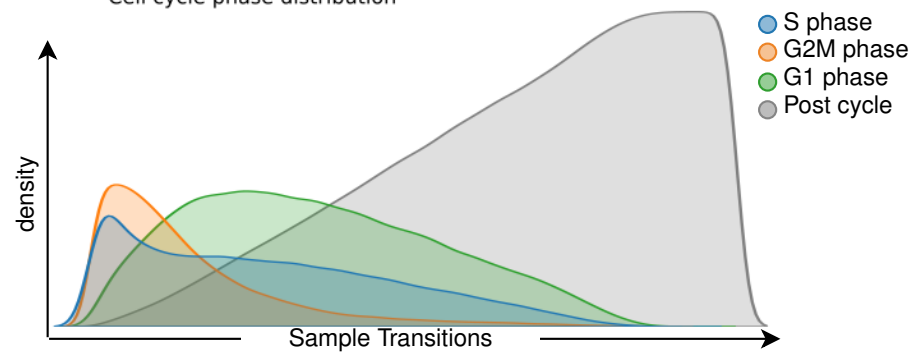

E

Average of Slingshot trajectories

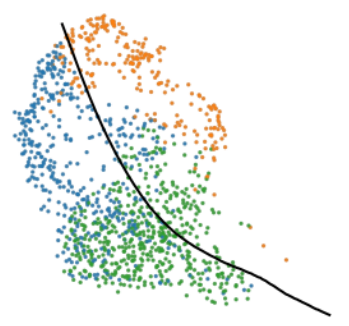

H
C
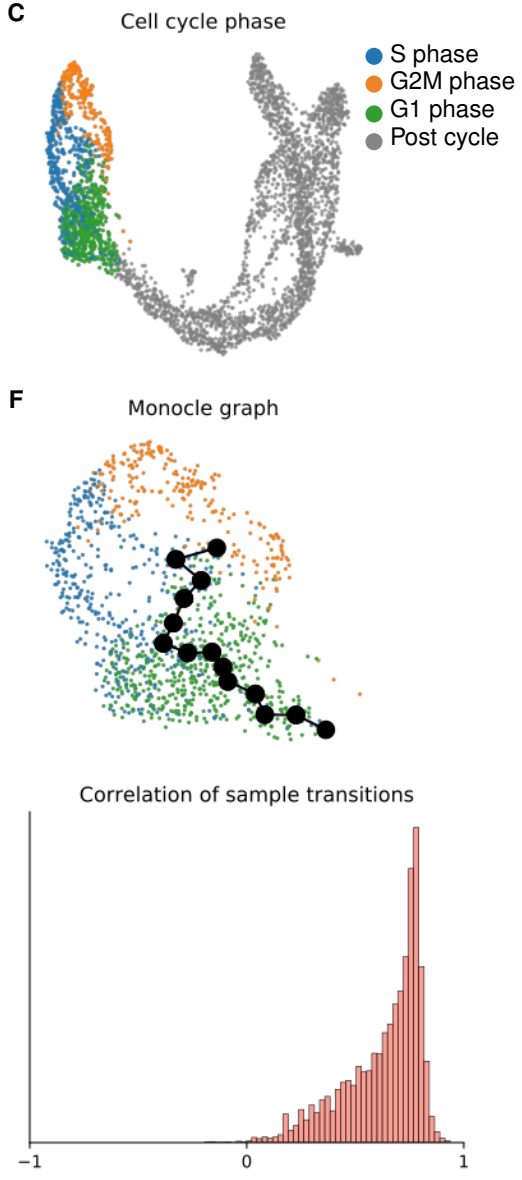

Figure 3: Reconstruction of interlaced cell cycling and bifurcated differentiation in pancreatic endocrinogenesis. (A) UMAP projection of pancreas scRNAseq data annotated with stages of differentiation. (B) Trajectories inferred by Cytopath and mean pseudotime per cell. (C) Estimated cell cycle phase assignment. (D-F) Cell cycle phase annotation and mean trajectory in the root region by Cytopath (D), Slingshot (E) and Monocle3 (F). (G) Cell cycle phase as a function of transition step of individual Cytopath simulations. (H) Spearman correlation of cell cycle phase with transition step of individual Cytopath simulations

\section{Reconstruction of bifurcating development of CD8+ T cells from scRNA seq time series}

We assessed the performance of Cytopath on a scRNAseq time-series dataset from CD8 T cell development in chronic LCMV infection [Cerletti et al., 2020]. In this infection model system T cells differentiate from early activated cells into exhausted and memory-like cells over a period of three weeks. Samples were collected at eight experimental time points after infection with LCMV and pooled to achieve four samples for sequencing, ultimately covering all stages of the process [Figure 4A]. Although these samples are heterogeneous snapshots of a spectrum of differentiation states at a particular time point they provide an approximate development coordinate. Starting from a population of cells at an early activated state, differentiation leads into the two distinct terminal states five days after LCMV infection. This differentiation is characterized by strong transcriptional changes and expression of different surface markers.

We identified the root and end points of the process computing the equilibrium of the Markov process as in [Manno et al., 2018] [Figure 4B-C]. The endpoints were validated by expression levels of known marker genes [Cerletti et al., 2020]. The exhausted endpoint showed high expression in co-inhibitory markers like CD39 (Entpd1), CD160 (Cd160) and PD-1 (Pdcdl). The memory-like endpoint had high expression of TCF1 (Tcf7) and IL-7R $(I l 7 r)$. Applying Cytopath to our data resulted in two trajectories leading from a shared starting region to the two expected endpoints. The two trajectories strongly overlap in the beginning of the process but then sharply diverge at a branching point [Figure $4 \mathrm{D}]$. 
A

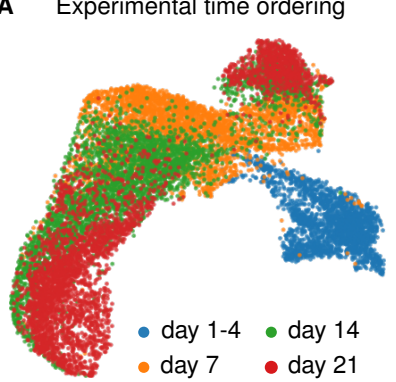

D Cytopath trajectories

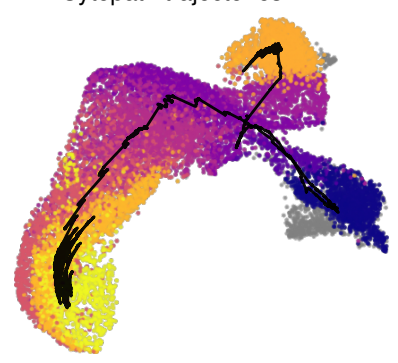

B Root state probability

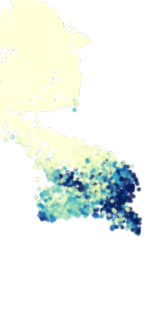

E

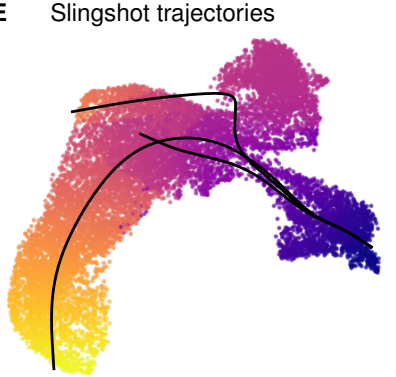

C Terminal state probability

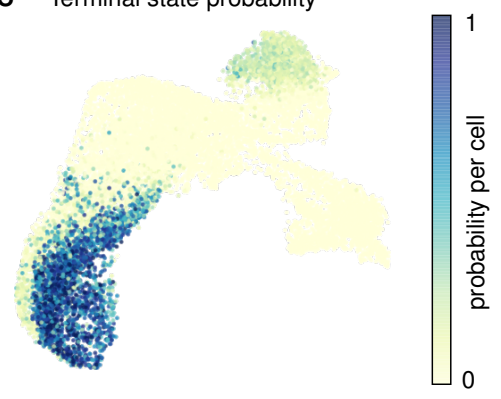

F Monocle graph

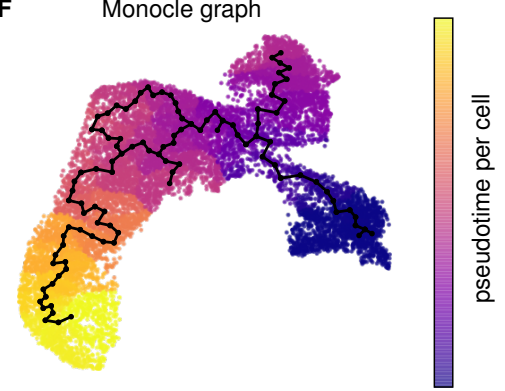

G

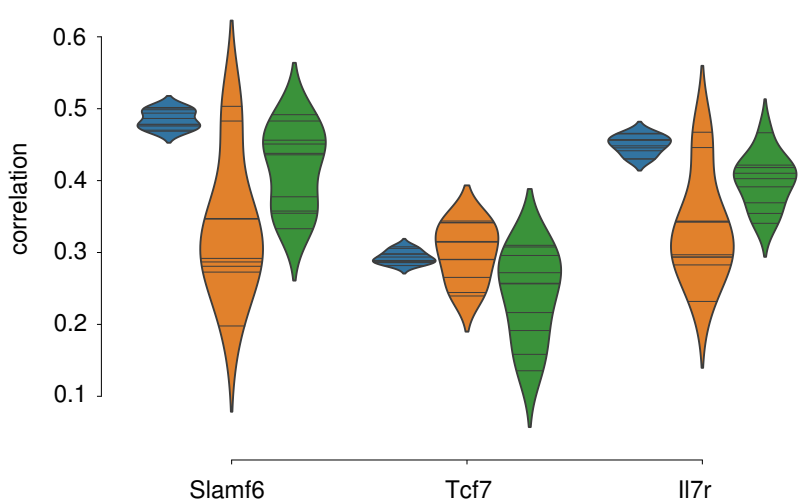

H

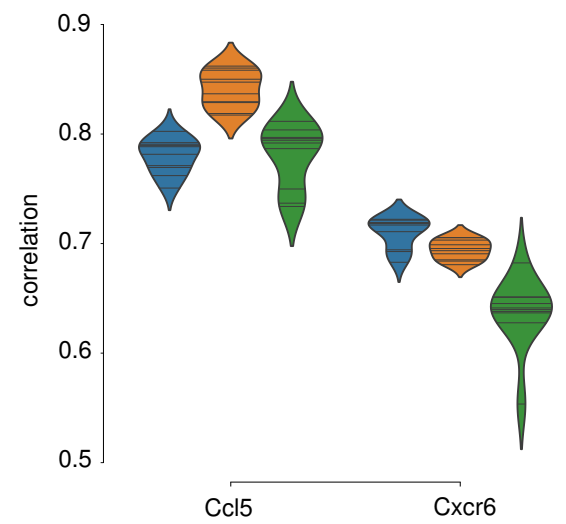

Figure 4: Reconstruction of bifurcating development of CD8+ $\mathbf{T}$ cells from scRNA seq time series. (A) Ordering of samples with respect to time of LCMV innoculation. (B-C) Probability estimated based on RNA velocity of the cell being (B) a root state (C) a termimal state (D-F) Trajectories inferred and mean pseudotime per cell by by Cytopath (D), Slingshot (E) Monocle3 (F). (G) Correlation of pseudotime estimated by each method with marker relevant to the Memory-like CD8+ T cell differentiation. (H) Correlation of pseudotime estimated by each method with marker relevant to the Exhausted CD8+ T cell differentiation.

Comparing the pseudotime estimates from Cytopath with the discrete experimental time labels from the samples showed high agreement of the two [Figure 5B]. The experimental time labels, corresponding roughly to the developmental coordinate, were ordered correctly and with very little overlap between the time-points. Despite the non-trivial structure of the UMAP projection, cells are ordered correctly. Although the development speed is occasionally abrupt the relative ordering is respected with the two endpoints having the highest pseudotime. This illustrates that the average trajectory produced by Cytopath respect the experimental time ordering of the cells [Figure 5D]. Slingshot found a trajectory towards the exhausted endpoint but did not infer a trajectory towards the memory-like endpoint [Figure 4E]. Instead, two spurious trajectory are generated ending prematurely. Monocle reconstructs the global structure of the data, but includes additional loops and branches within the exhaustion branch [Figure 4F]. Just like in Slingshot, pseudotime of the exhaustion branch is matching expectations, but the memory-like cell ordering does not recapture experimental time ordering. 
A

C

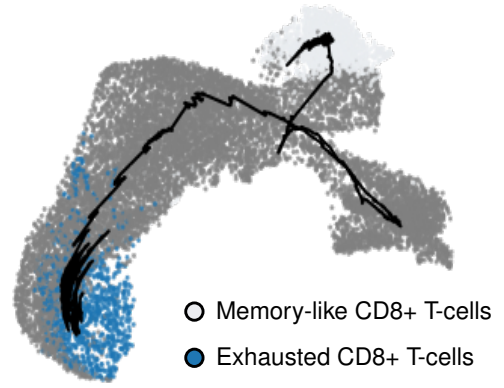

B

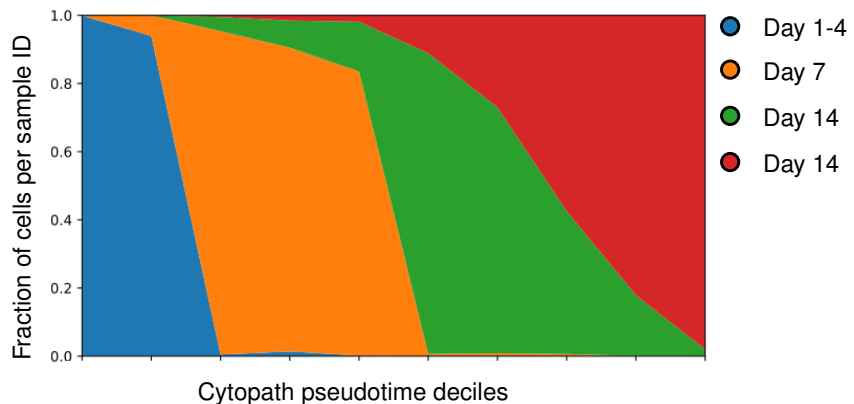

Cytopath pseudotime deciles

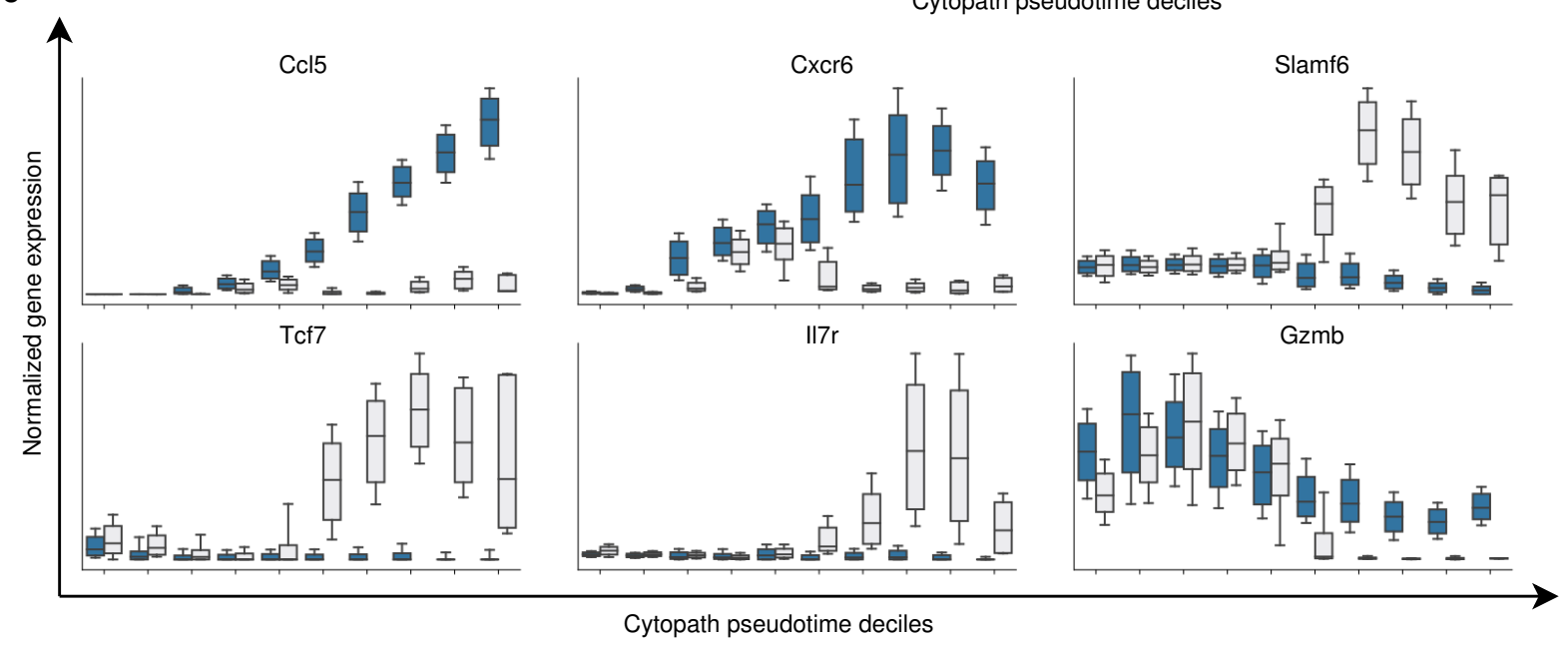

Figure 5: Cytopath trajectories identify exhaustion branch specific gene dynamics. (A) Biological identity of terminal states and trajectories inferred by Cytopath. (B) Fraction of cells from each experimental sample in each pseudotime decile. (C) Normalized expression of key marker genes in each pseudotime decile.

Further, we assessed the correlation of pseudotime estimiates with canonical gene expression markers of each the memory-like (Slamf6, Tcf7, Il7r) and exhaustion branch $(C c l 5, C x c r 8)$ [Figure 4G-H]. We observe that Cytopath achieves competitive to superior absolute performance and significantly lower variance according to this measure.

We further tested the validity of the average trajectories of Cytopath by the expression profiles of known lineage marker genes in the differentiation process. The chemokine receptor CXCR6 has been shown to mark exhausted T cells in chronic LCMV infection [Sandu et al., 2020]. The average expression of Cxcr6 increases in the trajectory towards the exhausted cluster right before the divergence of the two branches [Figure 5 C], indicating that the paths are indeed governed by the exhaustion process. Conversely T cell Factor 1 (TCF1) and the expression of its gene Tcf7 is an established marker of memory-like cells [Utzschneider et al., 2016]. Expression of this gene was increased in memory-like cells just after the cells started to diverge after the bifurcation point. Towards the memory-like endpoint at late time-points, $T c f 7$ expression is exclusive to the memory-like population. An additional observation is the high expression of Gzmb early both branches that drops off towards later timepoints [Figure 5C]. The expression of $G z m b$ is a shared feature of both branches and known to decrease in both branches as the infection progresses and expression is low towards late timepoints [Wherry et al., 2007].

In summary, we were able to reconstruct biologically relevant differentiation trajectories from a long-term time series dataset, in a more accurate and reproducible manner than baseline approaches not taking into account velocity information. We identified correct differentiation branches of CD8 T cells in chronic infection, demonstrated by correct ordering of the experimental time labels and expression levels of branch specific gene expression markers. 
A PREPRINT - DECEMBER 21, 2020

\section{Discussion}

Trajectory inference is a challenging task since scRNA seq data is noisy and until recently has been evaluated to achieve only static expression profile snapshots. Trajectory inference tools typically operate in low dimensional embeddings of scRNAseq data. Trajectory inference in lower dimensional spaces, especially two dimensional projections such as UMAP and t-SNE is particularly challenging for multifurcating datasets. Inclusion of directional transcriptional activity estimates from RNA velocity analyses is expected to achieve more precise and sensitive trajectory inference. With cytopath we present an approach that takes advantage of this information and we demonstrate superior capability to reconstruct complex differentiation hierarchies from scRNAseq data.

Transitions between cells are inferred on the basis of the high dimensional expression and velocity profiles. Since Cytopath is based upon transitions that use the full expression and velocity profiles of cells, it is less prone to projection artifacts distorting expression profile similarity. In addition, this approach specifically considers likely and discards unlikely transitions, and thereby is able to identify for instance cyclic trajectories in an apparently diffusely populated and isotropic region of expression space. Furthermore, these hidden transcriptional patterns are made apparent by its simulation based approach. RNA velocity based pseudotime and cell fate estimation based on analytical analysis of Markov chain properties, performed by scvelo and CellRank [Lange et al., 2020] do not readily present the user this information even if the pseudotemporal ordering estimated by these tools captures these patterns.

These properties render Cytopath an adequate tool to study complex differentiation processes such as the development of CD8 T cells in chronic infections. For this system several phenotypic populations and characteristic markers had been described before, but the connecting differentiation trajectories of those populations are a subject of ongoing research [Chen et al., 2019, Zander et al., 2019, Yao et al., 2019, Raju et al., 2020]. These studies provide evidence for branching in the development process, and only recently in conjunction with simulation based trajectory inference it was possible to resolve this event in more detail [Cerletti et al., 2020]. In addition to trajectory inference, Cytopath also returns the underlying simulations. Each simulation can be interpreted as a realisation of the biological process. Future work could expand on learning mechanistic differentiation models from these simulations.

In summary we expect simulation based trajectory inference approaches like Cytopath to enable sufficiently precise and unambiguous trajectory inference to achieve testable hypotheses to identify drivers and derive mathematical models of complex differentiation processes.

\section{Methods}

\section{Trajectory inference with Cytopath}

Cell clustering. Grouping of cell states reflecting biological similarity can be provided as input to Cytopath. By default Cytopath will perform clustering of cells using louvain.

Simulations. Simulations are initialized at random cell states selected uniformly within the defined root state cluster and consist of a fixed number of cell to cell transitions. At each step, a single transition from the current cell state is realised based on the cell to cell transition probability matrix $P$. The cell state $c_{i j}$ at step $i$ of simulation $j$ is selected randomly according to $P$ from the from the $k$ nearest neighbors of $c_{(i-1) j}$.

Trajectory inference. Simulations that terminate within defined terminal state clusters are considered for trajectory inference. Simulations are generated until a predefined number of unique simulations have been returned. Simulations are aligned using the fastdtw python package to achieve a trajectory. [Salvador and Chan, 2007] The median value of the expression profile of cells at each step of the aligned simulations is the coordinate of the trajectory at that step. By default a single trajectory is estimated for each root and terminal cluster pair.

Identification of compositional clusters. Trajectories are decomposed in compositional clusters from the set of clusters provided in the step cell clustering. For each step $i$ of the trajectory a fixed size set $M_{i}$ of neighboring cells are recovered using K-dimensional tree search. Cell clusters with cell representation larger than a threshold frequency of $\nu$ for at least one $M_{i}$ are considered a compositional cluster of the trajectory.

Alignment score. Alignment score of a cell with neighbors $K$ to step $i$ is the maximum of two scores. The score with respect to the trajectory segment from $i-1$ to $i, \xi_{i}$ is calculated as follows,

$$
\xi_{i}=\frac{1}{K} \sum_{k} \cos \left(\eta_{k}^{b}\right) \cdot \exp \left(\gamma_{k}\right)
$$

where $\eta$ is the cosine angle between the section of the trajectory and all possible transition partners $k$ of the cell. $\gamma$ is the cosine similarity between the velocity vector of the cell with the distance vector between the cell and it's neighbors. 
The score with respect to the trajectory segment from $i$ to $i+1, \tau_{i}$ is calculated similarly,

$$
\tau_{i}=\frac{1}{K} \sum_{k} \cos \left(\eta_{k}^{f}\right) \cdot \exp \left(\gamma_{k}\right)
$$

The alignment score of the cell with respect to step $i, d_{i}$ is

$$
d_{i}=\max \left(\xi_{i}, \tau_{i}\right)
$$

Pseudotime estimation. For each trajectory cells that have an alignment score greater than zero and also belong to a compositional cluster of the trajectory are assigned a pseudotime value with respect to the trajectory. Since a cell can align to multiple steps within a trajectory, the mean step value of a cell with respect to the trajectory is set to its pseudotime value.

\section{Datasets}

Dendate Gyrus. The dataset was obtained and processed using the following notebook (https://github.com/velocytoteam/velocyto-notebooks/blob/master/python/DentateGyrus.ipynb) [Manno et al., 2018].

Biological cell type annotation and RNA velocity derived root states were used to supervise trajectory inference for Cytopath, Slingshot and Monocle3 (root states only). Inference was peformed in the original T-SNE projection using default parameters for Slingshot and Monocle3. For Cytopath, in addition to the projection the original RNA velocity field was used to infer cell to cell transitions.

Pancreatic endocrinogenesis. The data was downloaded and processed using scvelo. RNA velocity was estimated using the deterministic model. Genes with less than ten combined spliced and unspliced counts were discarded. Expression was normalized by total count per cell. First fifty PCA components were used to perform the louvain clustering. Biological cell type annotation was used to supervise trajectory inference for all methods. Inference was performed in the original UMAP projection using default parameters for Slingshot and Monocle3. Cell cycle scoring was performed using scanpy.

CD8 development. Transgenic P14 CD8 T cells were sampled longitudinally during chronic infection with LCMV Clone-13 infection. The samples were acquired from four phases of the infection, namely activation (day 1-4), effector (day 7), early exhaustion (day 14) and late exhaustion (d21) and scRNAseq was performed using the 10x Genomics platform.

Read counts were realigned and sorted for spliced and unspliced counts using the analysis pipeline from velocyto [Manno et al., 2018]. Contaminating other cell types were removed from the dataset based on outliers in diffusion components. [Cerletti et al., 2020] RNA velocity was estimated using the deterministic model. Genes with less than ten combined spliced and unspliced counts were discarded. Expression was normalized by total count per cell. First fifty PCA components were used to perform the louvain clustering.

\section{Comparison of trajectory inference approaches}

Cluster containing cells with greater than 0.95 probability of being root states were designated as the root state in all datasets. Terminal states were defined as described in the original publications.

Spearman correlation of the pseudotime values generated by each method with the cell type cluster ordering for each biological lineage was used to compare the performance of the three methods. Kendall's tau was used to assess the correlation of marker expression with the estimated pseudotime.

Cytopath has been implemented as a python package and can be found at the following GitHub repository (https://github.com/aron0093/cytopath).

\section{References}

[Bergen et al., 2020] Bergen, V., Lange, M., Peidli, S., Wolf, F. A., and Theis, F. J. (2020). Generalizing rna velocity to transient cell states through dynamical modeling. Nature Biotechnology.

[Byrnes et al., 2018] Byrnes, L. E., Wong, D. M., Subramaniam, M., Meyer, N. P., Gilchrist, C. L., Knox, S. M., Tward, A. D., Ye, C. J., and Sneddon, J. B. (2018). Lineage dynamics of murine pancreatic development at single-cell resolution. Nature Communications, 9(1). 
[Cerletti et al., 2020] Cerletti, D., Sandu, I., Gupta, R., Oxenius, A., and Claassen, M. (2020). Fate trajectories of cd8+ t cells in chronic lcmv infection. unpublished.

[Chen et al., 2019] Chen, Z., Ji, Z., Ngiow, S. F., Manne, S., Cai, Z., Huang, A. C., Johnson, J., Staupe, R. P., Bengsch, B., Xu, C., Yu, S., Kurachi, M., Herati, R. S., Vella, L. A., Baxter, A. E., Wu, J. E., Khan, O., Beltra, J.-C., Giles, J. R., Stelekati, E., McLane, L. M., Lau, C. W., Yang, X., Berger, S. L., Vahedi, G., Ji, H., and Wherry, E. J. (2019). TCF-1-centered transcriptional network drives an effector versus exhausted CD8 $\mathrm{t}$ cell-fate decision. Immunity.

[Haghverdi et al., 2016] Haghverdi, L., Büttner, M., Wolf, F. A., Buettner, F., and Theis, F. J. (2016). Diffusion pseudotime robustly reconstructs lineage branching. Nature Methods, 13(10):845-848.

[Heinemann and Zenobi, 2011] Heinemann, M. and Zenobi, R. (2011). Single cell metabolomics. Current Opinion in Biotechnology, 22(1):26-31.

[Kulkarni et al., 2019] Kulkarni, A., Anderson, A. G., Merullo, D. P., and Konopka, G. (2019). Beyond bulk: a review of single cell transcriptomics methodologies and applications. Current Opinion in Biotechnology, 58:129-136.

[Lange et al., 2020] Lange, M., Bergen, V., Klein, M., Setty, M., Reuter, B., Bakhti, M., Lickert, H., Ansari, M., Schniering, J., Schiller, H. B., Pe'er, D., and Theis, F. J. (2020). CellRank for directed single-cell fate mapping. BiorXiv.

[Macnair et al., 2019] Macnair, W., Roditi, L. D. V., Ganscha, S., and Claassen, M. (2019). Tree-ensemble analysis assesses presence of multifurcations in single cell data. Molecular Systems Biology, 15(3).

[Manno et al., 2018] Manno, G. L., Soldatov, R., Zeisel, A., Braun, E., Hochgerner, H., Petukhov, V., Lidschreiber, K., Kastriti, M. E., Lönnerberg, P., Furlan, A., Fan, J., Borm, L. E., Liu, Z., van Bruggen, D., Guo, J., He, X., Barker, R., Sundström, E., Castelo-Branco, G., Cramer, P., Adameyko, I., Linnarsson, S., and Kharchenko, P. V. (2018). RNA velocity of single cells. Nature, 560(7719):494-498.

[Povinelli et al., 2018] Povinelli, B. J., Rodriguez-Meira, A., and Mead, A. J. (2018). Single cell analysis of normal and leukemic hematopoiesis. Molecular Aspects of Medicine, 59:85-94.

[Raju et al., 2020] Raju, S., Xia, Y., Daniel, B., Yost, K. E., Bradshaw, E., Tonc, E., Verbaro, D. J., Satpathy, A. T., and Egawa, T. (2020). Latent plasticity of effector-like exhausted CD8 $\mathrm{t}$ cells contributes to memory responses. BiorXive.

[Ren et al., 2018] Ren, X., Kang, B., and Zhang, Z. (2018). Understanding tumor ecosystems by single-cell sequencing: promises and limitations. Genome Biology, 19(1).

[Saelens et al., 2019] Saelens, W., Cannoodt, R., Todorov, H., and Saeys, Y. (2019). A comparison of single-cell trajectory inference methods. Nature Biotechnology.

[Salvador and Chan, 2007] Salvador, S. and Chan, P. (2007). Fastdtw: Toward accurate dynamic time warping in linear time and space. Armament and Technical Products.

[Sandu et al., 2020] Sandu, I., Cerletti, D., Oetiker, N., Borsa, M., Wagen, F., Spadafora, I., Welten, S. P., Stolz, U., Oxenius, A., and Claassen, M. (2020). Landscape of exhausted virus-specific CD8 T cells in chronic LCMV infection. Cell Reports, 32(8):108078.

[Street et al., 2018] Street, K., Risso, D., Fletcher, R. B., Das, D., Ngai, J., Yosef, N., Purdom, E., and Dudoit, S. (2018). Slingshot: cell lineage and pseudotime inference for single-cell transcriptomics. BMC Genomics, 19(1).

[Stubbington et al., 2017] Stubbington, M. J. T., Rozenblatt-Rosen, O., Regev, A., and Teichmann, S. A. (2017). Single-cell transcriptomics to explore the immune system in health and disease. Science, 358(6359):58-63.

[Trapnell et al., 2014] Trapnell, C., Cacchiarelli, D., Grimsby, J., Pokharel, P., Li, S., Morse, M., Lennon, N. J., Livak, K. J., Mikkelsen, T. S., and Rinn, J. L. (2014). The dynamics and regulators of cell fate decisions are revealed by pseudotemporal ordering of single cells. Nature Biotechnology, 32(4):381-386.

[Tritschler et al., 2019] Tritschler, S., Büttner, M., Fischer, D. S., Lange, M., Bergen, V., Lickert, H., and Theis, F. J. (2019). Concepts and limitations for learning developmental trajectories from single cell genomics. Development, 146(12):dev170506.

[Utzschneider et al., 2016] Utzschneider, D. T., Charmoy, M., Chennupati, V., Pousse, L., Ferreira, D. P., CalderonCopete, S., Danilo, M., Alfei, F., Hofmann, M., Wieland, D., Pradervand, S., Thimme, R., Zehn, D., and Held, W. (2016). T cell factor 1-expressing memory-like cd8 t cells sustain the immune response to chronic viral infections. Immunity, 45(2):415-427.

[Wherry et al., 2007] Wherry, E. J., Ha, S.-J., Kaech, S. M., Haining, W. N., Sarkar, S., Kalia, V., Subramaniam, S., Blattman, J. N., Barber, D. L., and Ahmed, R. (2007). Molecular signature of cd8+ t cell exhaustion during chronic viral infection. Immunity, 27(4):670-684.

[Wolf et al., 2018] Wolf, F. A., Angerer, P., and Theis, F. J. (2018). SCANPY: large-scale single-cell gene expression data analysis. Genome Biology, 19(1). 
[Wolf et al., 2019] Wolf, F. A., Hamey, F. K., Plass, M., Solana, J., Dahlin, J. S., Göttgens, B., Rajewsky, N., Simon, L., and Theis, F. J. (2019). PAGA: graph abstraction reconciles clustering with trajectory inference through a topology preserving map of single cells. Genome Biology, 20(1).

[Yang et al., 2019] Yang, L., George, J., and Wang, J. (2019). Deep profiling of cellular heterogeneity by emerging single-cell proteomic technologies. PROTEOMICS, 20(13):1900226.

[Yao et al., 2019] Yao, C., Sun, H.-W., Lacey, N. E., Ji, Y., Moseman, E. A., Shih, H.-Y., Heuston, E. F., Kirby, M., Anderson, S., Cheng, J., Khan, O., Handon, R., Reilley, J., Fioravanti, J., Hu, J., Gossa, S., Wherry, E. J., Gattinoni, L., McGavern, D. B., O'Shea, J. J., Schwartzberg, P. L., and Wu, T. (2019). Single-cell RNA-seq reveals TOX as a key regulator of CD8+ t cell persistence in chronic infection. Nature Immunology, 20(7):890-901.

[Zander et al., 2019] Zander, R., Schauder, D., Xin, G., Nguyen, C., Wu, X., Zajac, A., and Cui, W. (2019). CD4+ t cell help is required for the formation of a cytolytic CD8+ $\mathrm{t}$ cell subset that protects against chronic infection and cancer. Immunity, 51(6):1028-1042.e4.

[Zheng et al., 2017] Zheng, G. X. Y., Terry, J. M., Belgrader, P., Ryvkin, P., Bent, Z. W., Wilson, R., Ziraldo, S. B., Wheeler, T. D., McDermott, G. P., Zhu, J., Gregory, M. T., Shuga, J., Montesclaros, L., Underwood, J. G., Masquelier, D. A., Nishimura, S. Y., Schnall-Levin, M., Wyatt, P. W., Hindson, C. M., Bharadwaj, R., Wong, A., Ness, K. D., Beppu, L. W., Deeg, H. J., McFarland, C., Loeb, K. R., Valente, W. J., Ericson, N. G., Stevens, E. A., Radich, J. P., Mikkelsen, T. S., Hindson, B. J., and Bielas, J. H. (2017). Massively parallel digital transcriptional profiling of single cells. Nature Communications, 8(1). 


\section{Supplementary Figures}

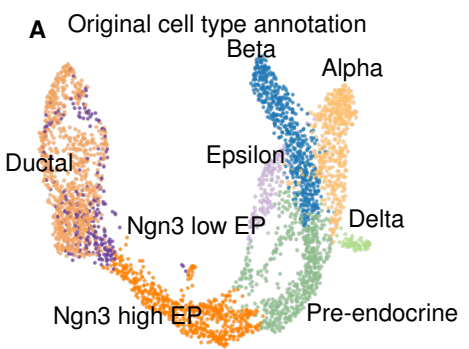

C Cytopath trajectories

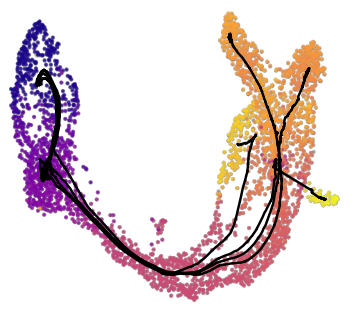

B

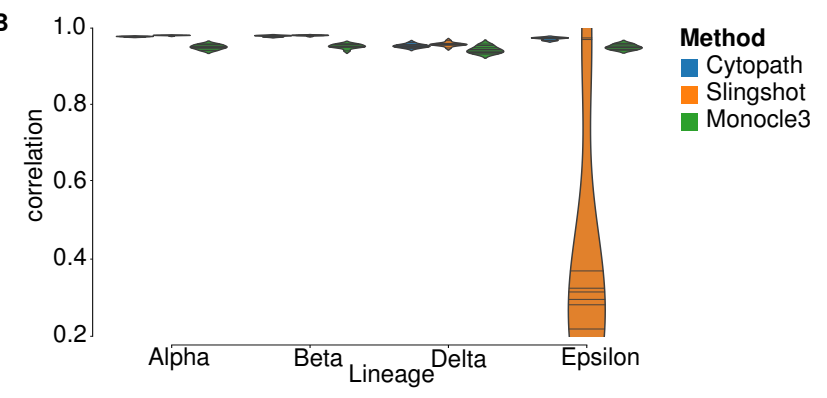

D

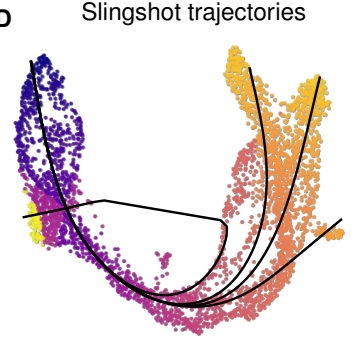

E Monocle graph

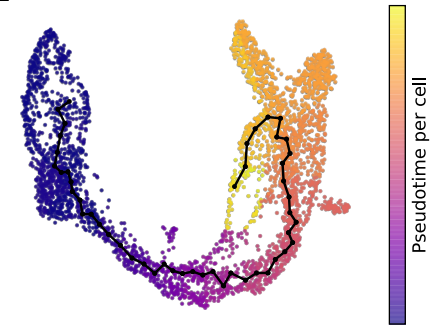

Figure S1: comparison of algorithms for pancreatic dataset

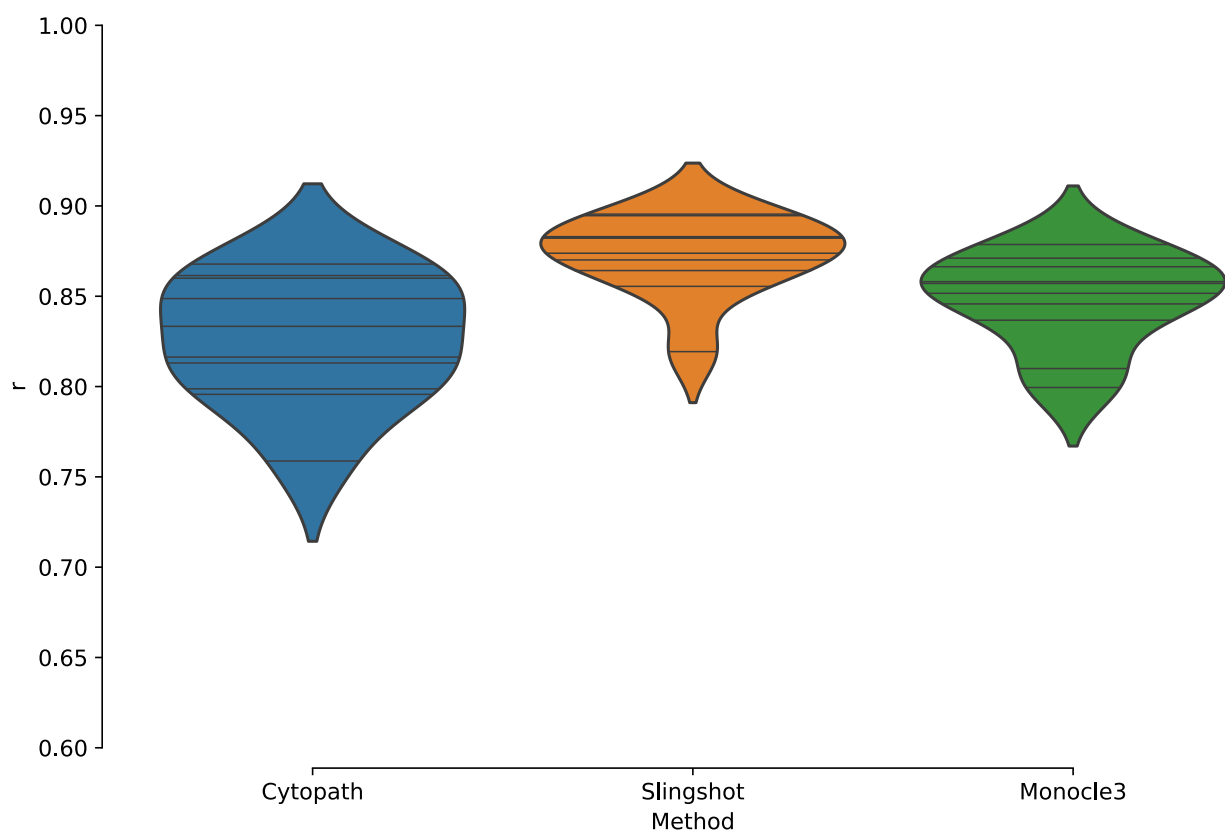

Figure S2: Correlation of experimental time ordering with average pseudotime estimated by Cytopath, Slingshot and Monocle3 in ten independent runs. 